\title{
Utilização de beacons em locais externos: Um estudo em um campus universitário no sul do Brasil
}

\author{
Marcelo Trindade Rebonatto, Cássio Eckstein, ${ }^{1}$, Cintia Sonale Rebonatto \\ 'Programa de Pós-Graduação em Computação Aplicada (PPGCA) Universidade de \\ Passo Fundo (UPF) - Passo Fundo, RS - Brasil \\ ²Programa de Pós-Graduação em Administração, Faculdade Meridional (IMED) - Passo \\ Fundo, RS - Brasil \\ cassiogc@gmail.com, rebonatto@upf.br, cintiasonale@gmail.com
}

\begin{abstract}
The emergence of beacons and Bluetooth Low Energy is already modifying and expanding the Internet of Things market. This project describes the development of a solution to gather contextual information about buildings and colleges on a university campus using beacons. The "Discover UPF" application was developed as a way to provide contextual information about the locations of a University's Campus. The implementation achieved its objectives, efficiently providing information to users while they are on the move on campus. The results indicate that the use of beacons in outdoor locations is possible and can be used in other applications. Some of the potentials and limitations of the technology have been clarified, as well as the expansion of their cases of possible future developments using beacon technology.
\end{abstract}

Resumo. O surgimento de beacons e Bluetooth Low Energy já está modificando e expandindo o mercado da Internet das Coisas. Este projeto descreve o desenvolvimento de uma solução para reunir informações contextuais sobre edificações e faculdades em um campus universitário usando beacons. Desenvolveu-se o aplicativo "Descubra UPF" como forma de fornecer informações contextuais sobre as localizações do Campus de uma Universidade. A implementação alcançou seus objetivos, fornecendo de forma eficiente informações aos usuários enquanto eles estão em movimento pelo campus. Os resultados apontam que o uso de balizas em locais ao ar livre é possível e pode ser usado em outras aplicações. Alguns dos potenciais $e$ limitações da tecnologia foram esclarecidos, bem como a expansão de seus casos de possíveis desenvolvimentos futuros usando a tecnologia beacon.

\section{Introdução}

Um tema discutido por pesquisadores da área de ciência da computação e que impulsiona um mercado com grande potencial é a Internet das Coisas (Internet of Things - IoT). Trata-se de uma perspectiva de um ambiente de interação com objetos 'inteligentes', por exemplo, uma geladeira que pode avisar o proprietário sobre os itens que estão em falta [Periyasamy and Dhanasekaran 2015]. O termo IoT pode ser usado para identificar três elementos distintos: a) o resultado da interconexão entre objetos inteligentes para estender as tecnologias da internet; b) as tecnologias utilizadas, como sensores, sistemas de comunicação entre máquinas; c) as tecnologias ou serviços que podem surgir a partir de IoT [Miorandi et al. 2012]. 
O surgimento da tecnologia Bluetooth Low Energy (BLE) pode ser considerado um dos impulsionadores do IoT, possibilitando a conexão entre dispositivos por meio da tecnologia Bluetooth, cujo diferencial é não possuir alto custo de energia e de hardware [Kuor-Hsin Chang 2014]. Com o surgimento do BLE, houve a criação da tecnologia beacon, que são dispositivos que emitem sinais contínuos[Kriz et al. 2016]. Esta tecnologia representa um dos pilares da IoT, e vem demonstrando o seu potencial em vários casos de sucesso, principalmente na área de vendas e marketing [Adarsh 2017].

Aplicações sensíveis ao contexto executadas em dispositivos móveis são uma realidade. Várias delas obtém informações a partir da localização atual do dispositivo (usuário), como equipamentos eletrônicos ou outros recursos físicos próximos ao dispositivo [Zaharakis and Komninos 2012]. Os beacons impulsionaram esses serviços, fornecendo um sinal de sua localização com baixo consumo de energia. Eles podem ser encontrados nos mais variados tipos de aplicações, desde o rastreamento de pessoas até o fornecimento informações nas mídias sobre produtos ou objetos em particular.

Os campus de universidades são, em geral, locais amplos e arborizados com vastas áreas verdes. Esses locais podem ser considerados similares a espaços verdes, oferecendo opções de lazer e atividades recreativas, envolvendo os habitantes de uma cidade e seu entorno [De Sousa 2003; Park and Kim 2019]. A circulação de pessoas da comunidade em geral nos campus universitários é uma realidade nas mais variadas regiões do Brasil. Esses locais possuem diversos prédios, com laboratórios e faculdades, contendo ambientes atrativos de serem visitados. Um problema para a comunidade não universitária é sua locomoção no campus, não conhecendo os recursos disponíveis nos prédios. Ademais, nem sempre há uma sinalização adequada, assim as características e funcionalidades dos prédios não conseguem ser corretamente exploradas.

Um elemento comum aos trabalhos com beacons é que mesmo aplicados em diversas áreas eles são em geral utilizados em ambientes fechados e em muitos casos associados a vendas. Nesse aspecto é que o presente estudo se diferencia dos demais, pois embora sejam encontrados em ambientes universitários, as aplicações envolvendo beacons em geral são centradas na rotina de professores e alunos. Este utiliza beacons com o objetivo de fornecer informações contextuais acerca da infraestrutura e funcionamento de prédios de um campus universitário. O objetivo é descrever uma solução aos usuários do campus universitário uma opção que facilite o reconhecimento das características e funcionalidades dos locais físicos, os quais comumente visitam sem perceber a quantidade de opções de entretenimento que podem ser exploradas.

\section{Comparativo entre beacons e outras tecnologias para IoT}

Outras tecnologias como identificação por rádio frequência (RFID), NFC ou códigos Quick Response (QR Code) podem ser utilizadas em aplicações IoT. Esta seção contém uma breve comparação dessas tecnologias em relação ao problema: permitir a obtenção de informações contextuais sobre o conteúdo de prédios em campus universitários.

O uso de NFC pode ser encontrado em aplicação direcionada a controle de frequência de alunos, sendo a mesma substituída por beacons devido ao seu baixo desempenho [Barapatre et al. 2017]. Na solução proposta neste artigo, o NFC não traria uma interface de uso confortável, uma vez que o usuário deveria aproximar seu smarthphone de uma tag NFC, para então obter as informações do local em questão.

A tecnologia de RFID permite a detecção de tags a diferentes distâncias. Porém, poucos smarthphones possuem suporte nativo ou possibilidade de adição de leitores 
RFID, sendo mais adaptada a leitura de tags RFID baseadas em NFC, o que limita muito a proximidade do usuário com a tag.

Um QR Code poderia ser impresso e fixado junto aos prédios, colocando neles uma informação acerca de onde acessar o conteúdo do prédio. Seria uma alternativa de menor custo, comparado ao uso de beacons. O problema dessa abordagem é a praticidade. Para o acesso a informação, o usuário deve se aproximar do QR Code para então o ler, processar e encontrar o link para as informações.

O GPS tem sido largamente utilizado por inúmeros tipos de sistemas, sendo uma tecnologia consolidada, utilizado para determinar a localização de um item específico ou indivíduo. Ele é empregado ainda no auxílio a navegação entre um ponto e outro. Como vantagens, destacam-se o alcance ilimitado, com cobertura mundial e a não geração de custos na utilização. Entre as desvantagens estão o consumo excessivo de energia durante o uso prolongado ou contínuo e a falta de precisão em locais fechados.

Entre as desvantagens dos beacons pode-se citar o alcance e o custo de aquisição. O alcance, considerado uma vantagem se comparado com NFC ou QR Code é uma desvantagem quando comparado com o GPS. O alcance médio dos beacons não ultrapassa 100 metros [Kosir 2016], devido ao fato que foram desenvolvidos para serem utilizados como uma maneira de receber informações baseadas em contexto [Paek et al. 2016]. O custo de aquisição é principal desvantagem, comparado com NFC ou QR Code e a gratuidade do GPS [Kosir 2016].

Entre as vantagens dos beacons em relação ao GPS reside o fato de que a busca continua não causa consumo excessivo de bateria. Outra vantagem em relação ao GPS é a facilidade de implementação. A criação de um sistema baseado no contexto de localização do usuário utilizando GPS pode ser uma tarefa desafiadora, pela necessidade de mapeamento geográfico das áreas e pela dificuldade de se determinar micro áreas uma vez que a precisão pode variar em até 10 metros. Isso pode ser ampliado se houver bloqueio dos sinais de satélite ocorridos pela presença de prédios [Posdorfer and Maalej 2016]. No caso dos beacons a sua implementação é simples, somente necessitando associar o Identificador Universal (UID) do beacon com o local. A principal vantagem do uso de beacons em relação a NFC ou QR Code é a facilidade para o usuário. Com NFC ou QR Code o usuário deve se deslocar e realizar ações com o smarthphone para então obter o conteúdo desejado. Com beacons, basta o usuário estar dentro do campo de cobertura que e a informação chegar diretamente, fornecendo-a de forma automática.

\section{Trabalhos relacionados}

Em [Barapatre et al. 2017] os autores propuseram o desenvolvimento de um aplicativo que utiliza beacons produzidos pela empresa Estimote com o protocolo Eddystone para registrar o comparecimento em aulas. O sistema anterior utilizava leitores de cartão Near Field Communication (NFC), porém este processo era lento no registro da frequência de estudantes. Os pesquisadores propuseram então o uso de um servidor em nuvem com uma integração no padrão Representational State Transfer (REST) na comunicação do servidor com o aplicativo.

Discutiu-se o uso de beacons das empresas Estimote, Radius Technologies e BKON, em ambientes educacionais na Universidade de Maiu (Hawaii) [Bhattacharya et al. 2017]. Nesse sentido, foi utilizado o protocolo Eddystone, especificamente o pacote Eddystone (EDI). O EDI permitiu a encriptação dos pacotes enviados, fazendo com que determinados beacons somente fossem detectados por usuários autorizados. O projeto 
teve por objetivo prover informações sobre menus da cafeteria, seções da biblioteca e atualizações de horários das aulas, fornecendo de uma URL para obter as informações.

O museu Guggenheim usa aplicação para exibir conteúdos aos usuários a partir da proximidade às obras de arte. Com seu smartphone e fone de ouvido o usuário circula pelo museu recebendo simultaneamente informações relativas as obras de arte, a estrutura e história do museu. As informações são textos, imagens e vídeos, até uma breve história por trás de cada obra. Após um ano da utilização, notou-se que o uso do aplicativo ultrapassou as formas tradicionais de pesquisas, como telas touchscreen e listas digitais das obras espalhadas pelo museu [GMF 2017].

Beacons foram adotados como instrumentos de posicionamento em locais fechados para uma loja de varejo na Grécia [Triantafyllou et al. 2017]. Foram distribuídos aproximadamente 90 beacons nos dois andares da loja, para que fosse possível realizar a triangulação do usuário, obtendo a sua localização. O aplicativo permitia detectar e registrar a localização do usuário em um intervalo específico de tempo. A partir dos dados coletados, foram utilizadas técnicas de machine learning para detectar locais e produtos mais visitados pelo cliente, fornecendo conteúdos direcionados ao perfil de cada um.

A utilização de beacons para o marketing de proximidade foi apresentado em [Allurwar et al. 2016]. Nesse estudo foram apresentadas as possíveis implementações da tecnologia como: notificação de produtos disponíveis para venda próximos do consumidor, pagamentos sem a interação com funcionários, recomendação de produtos com base na estimativa do tempo em que o cliente permanece próximo a um produto, informações sobre produtos similares e oferta de cupons de desconto. Como resultado, a pesquisa permitiu verificar que com o uso dessa tecnologia foi possível melhorar a experiência dos usuários e, por consequência fidelizar um número maior de clientes.

É possível perceber que beacons tem sido utilizados nos mais variados meios. Em alguns casos, aliados a técnicas de inteligência artificial, o que pode expandir as suas possibilidades de aplicações. Aplicações em ambientes universitários como em [Barapatre et al. 2017] mostram uma tendência de que as novas tecnologias sejam empregadas em ambientes universitários se não antes, junto com outras aplicações.

Um fato comum aos trabalhos apresentados é que em todos, o local de aplicação dos beacons ocorreu em ambientes fechados, possibilitando informações contextuais aos usuários de objetos ou produtos. Não foram encontradas aplicações de beacons em ambientes ao ar livre (abertos), nem tampouco informando serviços que funcionam em prédios, sobre as características de laboratórios ou cursos em funcionamento.

O presente trabalho utiliza beacons com o objetivo de fornecer informações contextuais acerca da infra-estrutura e funcionamento de prédios em campus . Sua área de aplicação são ambientes universitários, os mesmos usados em [Barapatre et al. 2017] e [Bhattacharya et al. 2017]. Porém, o objetivo final se assemelha a outros trabalhos [Allurwar et al. 2016; Ferreira Paz et al. 2017; GMF 2017; Triantafyllou et al. 2017], porém aplicado em ambientes abertos e não direcionado a vendas.

\section{Material e métodos}

A fim de validar o uso de beacons para fornecer informações contextuais sobre o conteúdo de prédios em campus universitários (laboratórios, cursos, serviços), desenvolveu-se um aplicativo móvel que obtém informações sensíveis ao contexto a partir da localização do usuário e proximidade com beacons [Eckstein and Rebonatto 
2018]. Os beacons utilizados são da empresa Estimote, por ser citada como empresa referência de beacons no mercado e usados em casos de sucesso [GMF 2017], [Barapatre et al. 2017]. A linguagem de programação escolhida foi o Java, acompanhada do Android Software Development Kit. Optou-se pela mesma por ser considerada uma linguagem de programação adequada ao desenvolvimento de aplicativos Android. Adotou-se o Android Studio como Integrated Development Enviroment (IDE) por ser a oficial disponibilizada pela Google, para o desenvolvimento de aplicativos que executam no SO Android. Ela possui várias ferramentas integradas, como o Gradle usado para gerenciamento de pacotes e possibilita o uso de emuladores de versões do Android para testes e validações.

Na detecção dos beacons e na recepção de anúncios foi escolhida a biblioteca Nearby [Google 2018a] em particular as seções Nearby Messages e Nearby Notifications. A Nearby proporciona uma integração rápida na detecção de beacons e na estimativa da distância do dispositivo móvel. Os beacons precisam ser registrados no Google Beacon Dashboard por meio do Beacon Tools, onde são cadastrados o anúncio e dados básicos em formato JavaScript Object Notation (JSON). Foi usada a Proximity API na conexão com o dashboard, para obter as informações [Google 2017].

A potência de emissão foi configurada nos beacons por meio do aplicativo da Estimote. As opções da potência de emissão variam de um 1 a 70 metros sendo definido 40 metros para o raio, aproximadamente. Não há precisão neste valor, em virtude de serem emissões de onda de rádio, variando conforme os fatores externos. Os beacons foram configurados para emitirem o pacote Eddystone-UID, utilizado pelo Nearby. Um estudo aprofundado sobre os limites e taxas de transmissão, assim como mecanismos de emissão dos sinais dos beacons extrapola os limites desse texto. Maiores informações nesse sentido podem ser encontradas em [Castillo-Cara et al. 2017].

Para o armazenamento dos dados e das imagens o Firebase foi adotado [Google 2018b]. Trata-se de uma ferramenta que possui diferentes opções de recursos. Foram utilizados o Firestore e o Storage, onde o primeiro é um banco de dados (BD) NoSQL orientado a documentos. O segundo é um sistema de arquivos utilizado para imagens. Para a execução dos vídeos a Player API do YouTube foi escolhida, pois disponibiliza um player semelhante ao utilizado pelo aplicativo [Google 2018c]. Para a obtenção de informações referentes aos vídeos foi usada a Data API do YouTube, que realiza chamadas REST na obtenção das informações [Google 2018d].

Um BD para armazenar as informações do projeto foi modelado e implementado, sendo dividido em três partes: dados básicos, conteúdo e imagens. Os dados básicos são necessários para a listagem dos locais no momento da detecção de um beacon e desta forma é obrigatório o seu preenchimento. A informação essencial é o UID associado ao local, bem como sua localização (latitude e longitude). Armazenou-se o conteúdo em um BD NoSQL orientado a documentos devido a: I) possibilidade de armazenar todas as informações de um beacon em um documento, facilitando o manuseio das informações no código; II) não haver relacionamento entre nenhum dos dados ou campos e, III) flexibilidade de criar um documento sem especificação de formato. Essas informações associadas aos beacons agrupam informações como arquivos utilizados para mostrar o conteúdo relacionado ao local, listas de imagens e vídeos. No armazenamento das imagens é criada uma pasta para cada beacon, com o nome sendo o UID do respectivo beacon. O nome dos arquivos inseridos nas pastas é indiferente, pois os mesmos são associados aos elementos das listas. 


\section{Resultados e Discussões}

O aplicativo foi validado por meio de um caso de estudo, fornecendo informações de um campus universitário com aproximadamente 400 hectares, sendo cerca de 120.000 $\mathrm{m}^{2}$ de área urbanizada. A área está dividida entre áreas verdes com aproximadamente 11 hectares, 10 hectares em área de preservação permanente, 92 prédios onde funcionam 60 cursos de graduação, 15 programas de stricto-sensu e 230 laboratórios.

Montou-se dentro do campus um ambiente de testes com dois prédios físicos. Ele foi criado com uso de três beacons (um dos prédios possui duas entradas, sendo os beacons colocados próximos a cada uma delas). Estes prédios são vizinhos, fazendo parte da região central do campus. O aplicativo foi validado com um smartphone da marca Xiaomi modelo Mi 5. O mesmo dispositivo serviu para testar no SO Android em diferentes versões. Há suporte a configuração do idioma de interface com o usuários no aplicativo, sendo os disponíveis são o Português (Brasil) e Inglês (Estados Unidos).

A solução com beacons foi testada após o aplicativo ser finalizado pela equipe de desenvolvimento do projeto e voluntários. Ele conseguiu atender as necessidades relatando de forma correta as informações sobre os locais a medida em que os usuários se locomoviam pelo campus. As atualizações das distâncias dos beacons em relação a posição atual do usuário foram corretamente computadas e atualizadas, mostrando aos usuários as informações referentes ao prédio mais próximo de sua localização e os demais, dentro da área de alcance dos beacons. A medida em que o usuário extrapola a distância de alcance configurado para os beacons, os locais vão deixando de ser mostrados no aplicativo, bem como a lista de locais é atualizada em tempo de execução.

\subsection{Interface do usuário e detecção dos beacons}

A Graphical User Interface consiste em duas áreas principais: uma área de navegação e outra de informações. Na Figura1 é possível visualizar algumas screenshots do aplicativo. A área de navegação possui quatro telas: listagem dos beacons (Figura 1(a) a esquerda), mapa, histórico e configurações. Na tela de listagem são descritos os locais detectados via beacons. Na visualização da lista há uma imagem referente ao local em que o beacon se encontra, o nome abreviado e o nome completo do local. A partir dessa lista, o usuário pode selecionar um dos locais, acessando a área de informações. Na segunda tela há um mapa demonstrando a posição atual do usuário assim como a localização geográfica dos beacons detectados. A tela do histórico caracteriza-se por possuir uma lista de ações exercidas pelo usuário, bem com como ações do sistema. Um menu, na parte inferior da área de navegação permite a troca entre as telas.

Após um elemento da lista ser selecionado, o usuário é redirecionado a área de informações (Figura 1(b) a direita), que mostra as informações sobre o local selecionado. Nesta área há três telas: informações gerais, imagens e vídeos. A tela de imagens é uma lista das imagens disponíveis com thumbnails e uma descrição. Ao selecionar uma imagem a mesma pode ser visualizada em tela cheia, onde é possível navegar pela lista em uma barra inferior. Na tela dos vídeos, cada item da lista possui uma thumbnail do vídeo, assim como o título e sua duração. Ao clicar no vídeo o mesmo é aberto e executado em tela cheia, utilizando-se o player do YouTube.

Para a detecção dos beacons é necessária a ativação do bluetooh no smartphone. Uma tela de introdução para o aplicativo foi criada, exibida somente na primeira execução do aplicativo. Informações sobre o aplicativo e o seu objetivo são exibidas com uma requisição da permissão do uso do bluetooth e uma explicação da necessidade. 


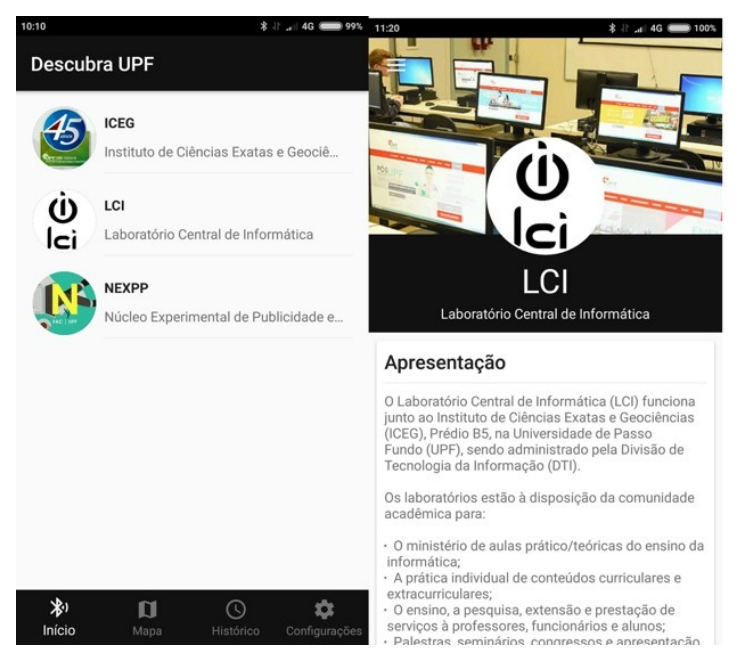

Figura 1: Interface com o usuário do aplicativo DescubraUPF

Caso o usuário não possua o aplicativo instalado, porém está com seu bluetooth ativado, uma notificação de anúncio do aplicativo é emitida ao dispositivo móvel. A notificação é uma das formas de comunicação com beacons e objetiva informar o usuário que foram encontrados beacons registrados e, consequentemente, ele está próximo de locais no campus que foram mapeados. Dessa forma, um usuário que chega ao campus pode ficar sabendo que há um aplicativo para auxiliá-lo na visita. A notificação aparecerá decorridos aproximadamente 35 segundos após o usuário entrar raio de alcance de algum beacon. Caso o aplicativo seja colocado em segundo plano, decorridos cinco (5) minutos, uma notificação é ser lançada para informar ao usuário que ainda podem ser visualizadas informações sobre locais. Não ocorrem notificações duplicadas do mesmo BLE e no momento em que o usuário deixa a área de alcance do beacon, ocorre a remoção da notificação. Uma solução para o problema de sobrecarga de notificações é o uso de apenas um beacon para o anúncio, posicionado no pórtico de entrada do Campus. Assim, apenas um beacon irá emitir notificações.

\subsection{Funcionamento do aplicativo}

Quando o usuário se locomove pela extensão do campus universitário com o seu dispositivo móvel, o aplicativo rastreia os beacons visíveis (dentro de sua área de alcance) próximos. Quando um beacon é encontrado, o aplicativo consulta as informações armazenadas e as disponibiliza ao usuário de acordo com o local encontrado. Um diagrama de atividades do processo de detecção dos beacons e atualização das informações pode ser visualizada na Figura 2.

A busca pelos beacons e as alterações de distância são ações em que não há interação como o usuário, sendo realizadas ininterruptamente. São usadas Nearby Messages para pesquisar os beacons que estejam emitindo os sinais. Quando algum deles é detectado, o aplicativo se conecta com o Proximity Beacon API para verificar se ele faz parte do projeto, retornando as informações básicas cadastradas sendo o local associado adicionado à lista de prédios/laboratórios próximos. Qualquer alteração de distância do smartphone em relação aos beacons, calculada pelo Nearby, faz com que a lista de locais detectados seja reordenada, deixando o mais próximo do dispositivo móvel no topo. Se o dispositivo perder contato com algum beacon, o mesmo é removido da lista. 


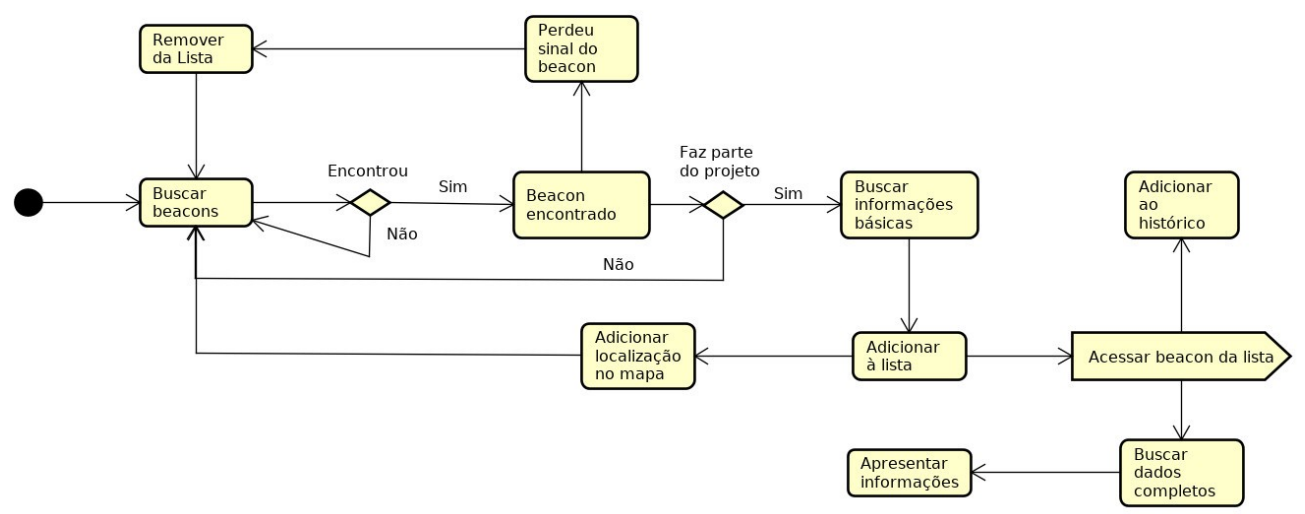

Figura 2: Diagrama de atividade da detecção dos beacons, contemplando parte das ações a partir da intervenção do usuário

Nota-se que na primeira busca por beacons após a instalação, o tempo decorrido para encontrar algum foi de aproximadamente cinco (5) segundos e nas execuções seguintes de dois (2) segundos. Isso ocorre pelo fato do Nearby utilizar cache para salvar os dados utilizados. A atualização das distâncias ocorre sem intervalos, o Nearby notifica o aplicativo de alterações.

Independente da ação, descoberta ou perda de sinal de algum beacon, o mapa será atualizado com a localização geográfica dos beacons. Ao selecionar algum dos locais mapeados por beacons e detectados, o aplicativo busca na base de dados as informações descritivas referentes ao local. Se o usuário desejar visualizar as fotos e vídeos disponibilizadas ele pode acessá-las.

\subsection{Comunicação para obtenção de informações e videos}

Ao ser selecionado um item da lista, é realizada uma consulta ao Firestore utilizando o UID do beacon para buscar as informações do local. Os nomes dos arquivos salvos no Storage e os identificadores dos vídeos do YouTube também são recuperados. Ao acessar as imagens são mostraas as da pasta relacionada ao beacon. Um diagrama de sequencia (Figura 3) demonstra o funcionamento para a obtenção dos dados no Firestore e no Storage assim como nos serviços do YouTube Player e Data APIs.

Ao acessar a tela dos vídeos, utilizam-se os identificadores para carregá-los no aplicativo com as APIs do YouTube. A criação da lista com os vídeos disponíveis, é realizada utilizado a Player API para a busca das thumbnails. Esta API não disponibiliza os dados do vídeo, como tempo de duração, título, descrição e dados analíticos. Assim, houve a opção pela Data API, onde são realizadas requisições REST por meio do uso dos identificadores dos vídeos a fim de obter estas informações e incorporá-las à listagem.

As fotos podem demorar até cinco (5) segundos para serem carregadas na primeira execução e nas seguintes em menos de um (1) segundo. Isso ocorre devido a utilização de cache contido no Firebase. O conteúdo de fotos e vídeos são recebidos e visualizados via streaming, para não impedir o acesso as demais informações e não consumir acesso de dados a internet do usuário se o mesmo não desejar visualizá-los.

\section{Considerações finais}

Este artigo apresentou uma solução para a localização de usuários em um campus universitário com uso de Ultra Low Power Bluetooth Technology, por meio de 
dispositivos conhecidos como beacons. A solução foi projetada, implementada e validada. Apresentou-se adicionalmente uma relação de trabalhos relacionados, completando com um breve descrição dos tipos, aplicações e tecnologias empregados em conjunto com beacons.

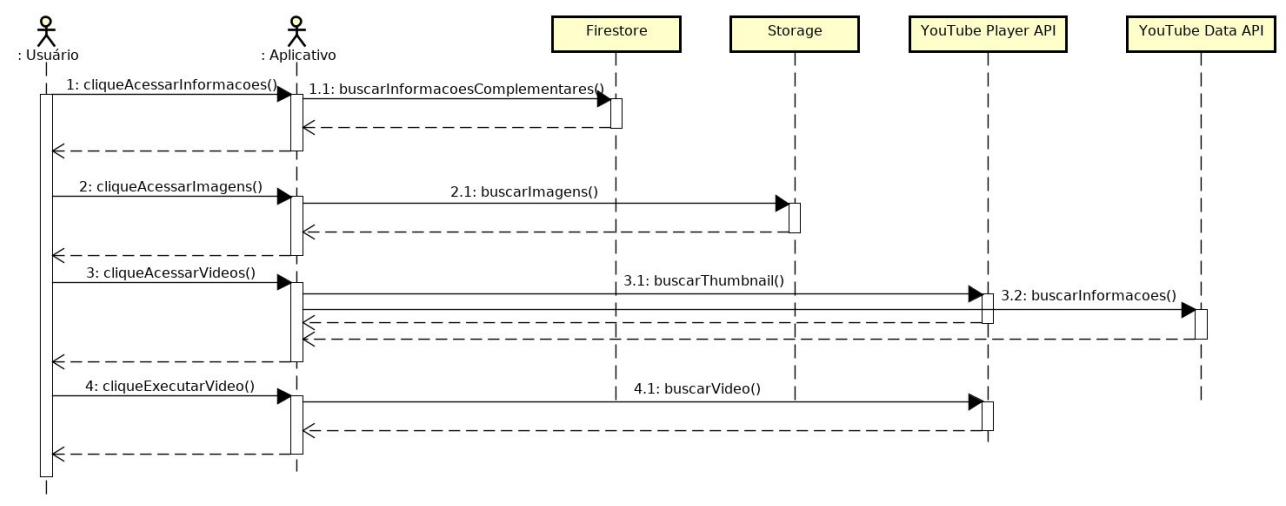

Figura 3: Diagrama de sequência da comunicação entre o aplicativo desenvolvidos e serviços externos na nuvem

A partir dos testes realizados com o aplicativo, pode-se afirmar que a tecnologia beacon se mostrou uma alternativa viável e interessante para a solução do problema de localização em ambientes externos, assim como a obtenção de informações contextuais. Torna-se possível afirmar ainda que o aplicativo realmente facilita a obtenção de informações contextuais e, com certeza, é possível que os beacons sejam usados nos mais diversos contextos, como nesse caso em ambientes não fechados.

Uma possível evolução deste estudo seria a criação de um sistema similar ao desenvolvido em [Barapatre et al. 2017], ou seja, a criação de um sistema automático de presenças. Isto é, um aplicativo que permita detectar a sala de aula na qual o aluno se encontra e quanto tempo o mesmo permaneceu nesta localização, determinando a sua assiduidade. Além do controle da frequência, poderia ser realizado um controle do fluxo de pessoas (alunos) nos locais de um campus. Esse controle de pessoas poderia beneficiar o registro em atividades diversas como palestras e eventos, bem como a concentração em áreas como paradas de ônibus. Dessa forma, os beacons poderiam ampliar as perspectivas dos Smart Campus.

\section{References}

Adarsh, M. (2017). 11 Incredible beacon use cases from 2017 every marketer should know in 2018. . https://blog.beaconstac.com/2017/12/11-incredible-beacon-usecases-from-2017-every-marketer-should-know-before-2018/.

Allurwar, N., Nawale, B. and Patel, S. (2016). Beacon for Proximity Target Marketing. International Journal Of Engineering And Computer Science, v. 5, n. 5, p. 1635916365.

Barapatre, A. S., Shelake, V. A., Pawar, G. Y. and D.R., A. (2017). Smart College System using IoT BLE Beacons. Ijarcce, v. 6, n. 4, p. 438-442.

Bhattacharya, D., Canul, M. and Knight, S. (2017). Case Study : Impact of the Physical Web and BLE Beacons. In Proceedings of Hawaii International Conference on System Sciences.

Castillo-Cara, M., Lovón-Melgarejo, J., Bravo-Rocca, G., Orozco-Barbosa, L. and García- 
Varea, I. (jun 2017). An Empirical Study of the Transmission Power Setting for BluetoothBased Indoor Localization Mechanisms. Sensors, v. 17, n. 6, p. 1318.

De Sousa, C. A. (feb 2003). Turning brownfields into green space in the City of Toronto. Landscape and Urban Planning, v. 62, n. 4, p. 181-198.

Eckstein, C. G. and Rebonatto, M. T. (2018). Descubra UPF: uma solução para facilitar o acesso a informações contextuais usando beacons. Universidade de Passo Fundo.

Ferreira Paz, L., Maran, V., Machado, A. and Augustin, I. (2017). MECA: Mobile System Support for Brazilian Community Health Agents Program Based on Context-Awareness. IEEE Latin America Transactions, v. 15, n. 8, p. 1547-1555.

Google (2017). Proximity Beacon API. . https://developers.google.com/beacons/proximity/guides.

Google (2018a). Nearby. . https://developers.google.com/nearby/.

Google (2018b). Firebase. . https://firebase.google.com/.

Google (2018c). YouTube Android Player API. . https://developers.google.com/youtube/android/player/.

Google (2018d). YouTube Data API. . https://developers.google.com/youtube/v3/.

Kosir, D. (2016). Location-Based Technology for Mobile Apps: Beacons vs. GPS vs. WiFi. . https://clearbridgemobile.com/location-based-technology-for-mobile-apps-beacons-vs-gpsvs-wifi/.

Kriz, P., Maly, F. and Kozel, T. (2016). Improving Indoor Localization Using Bluetooth Low Energy Beacons. Mobile Information Systems, v. 2016.

Kuor-Hsin Chang (2014). Bluetooth: a viable solution for IoT? [Industry Perspectives]. IEEE Wireless Communications, v. 21, n. 6, p. 6-7.

Miorandi, D., Sicari, S., De Pellegrini, F. and Chlamtac, I. (2012). Internet of things: Vision, applications and research challenges. Ad Hoc Networks, v. 10, n. 7, p. 1497-1516.

Paek, J., Ko, J. and Shin, H. (2016). A Measurement Study of BLE iBeacon and Geometric Adjustment Scheme for Indoor Location-Based Mobile Applications. Mobile Information Systems, v. 2016.

Park, J. and Kim, J. (jan 2019). Economic impacts of a linear urban park on local businesses: The case of Gyeongui Line Forest Park in Seoul. Landscape and Urban Planning, v. 181, p. 139-147.

Periyasamy, M. and Dhanasekaran, R. (apr 2015). Evaluation of performance of UHF passive RFID system in metal and liquid environment. In 2015 International Conference on Communications and Signal Processing (ICCSP). . IEEE. http://ieeexplore.ieee.org/document/7322921/.

Posdorfer, W. and Maalej, W. (2016). Towards Context-aware Surveys Using Bluetooth Beacons. Procedia Computer Science, v. 83, n. Ant, p. 42-49.

The Guggenheim Museums and Foundation (2017). The icon of modern art puts Estimote beacons on display. . http://blog.estimote.com/post/157200820650/the-icon-of-modern-artputs-estimote-beacons-on.

Triantafyllou, S., Koutsokera, L., Stavrou, V. and Griva, A. (2017). Enhance shopping experience and support decision making leveraging BLE beacons in a grocery retail store. In Proceedings of 14th Management Science and Technology Student Conference.

Zaharakis, I. and Komninos, A. (apr 2012). Ubiquitous computing multidisciplinary endeavour. IEEE Latin America Transactions, v. 10, n. 3, p. 1850-1852. 